\title{
Raman spectrum: A potential biomarker for embryo assessment during in vitro fertilization
}

\author{
JIAYI DING ${ }^{1}$, TIAN XU ${ }^{2}$, XIAOFANG TAN $^{1}$, HUA JIN $^{1}$, JUN SHAO $^{1}$ and HAIBO LI \\ ${ }^{1}$ Department of Clinical Laboratory Medicine, Nantong Maternity and Child Health Hospital, Nantong, Jiangsu 226018; \\ ${ }^{2}$ Department of Physics, School of Science, Nantong University, Nantong, Jiangsu 226019, P.R. China
}

Received June 27, 2016; Accepted January 26, 2017

DOI: $10.3892 /$ etm.2017.4160

\begin{abstract}
The aim of the study was to investigate whether Raman spectrum is consistent with the morphological scoring of the embryo of day 3 during in vitro fertilization (IVF). The spent culture media of embryo of day 3 from 10 patients were collected and analyzed. The samples were analyzed using Raman spectroscopy and graded according to the standard embryo scoring system simultaneously. Data showed that the Raman spectra obtained from the droplet of media were useful, as they can act as the characteristic signature for protein and amino acids. The Raman biospectroscopybased metabonomics profiling of spent media was consistent with the result of conventional morphological evaluation. In conclusion, this technology offers great potential for the development of tools allowing rapid non-invasive assessment of the quality of embryo of day 3 during IVF.
\end{abstract}

\section{Introduction}

Infertility is a serious public health issue, estimated to affect $15 \%$ of the reproductive aged population. It has gained much attention worldwide. In vitro fertilization (IVF) has appeared as a powerful solution in the aspect of infertility treatment (1). The process of IVF involves monitoring and stimulating a woman's ovulatory process and further removing an ovum or ova (egg or eggs) from the woman's ovaries for IVF with sperm in a laboratory. The fertilized egg (zygote) is cultured for 2 to 6 days in a growth medium and then implanted in the woman's uterus with intention of establishing a successful pregnancy (2). Accordingly, morphological assessment was the primary method used in the fertility clinics to select in vitro generated embryo(s) for the transfer into the uterus (3). However, it was widely known that the correlation between

Correspondence to: Dr Haibo Li, Department of Clinical Laboratory Medicine, Nantong Maternity and Child Health Hospital, 399 Century Avenue, Nantong, Jiangsu 226018, P.R. China

E-mail: 1i_haibo9@163.com

Key words: Raman spectrum, embryo, in vitro fertilization, biomarker embryo morphology and implantation potential is also elusive (4). Indeed, embryo morphological grading methods mainly depends on the observer experience and the assessment criteria is rather subjective. As a milestone technique in reproductive medicine, the first time-lapse microscopy system for IVF was approved in 2009 for clinical use. Embryo morphokinetic scoring system has been shown to further improve the rates of pregnancy (5). The images were compiled using specialized software to create a time-lapse sequence of embryo development. Consequently, the study negated the need for the embryologist to remove the embryos from the incubator for morphological assessment. Additionally, widely using this technique also addresses some of the encountered obstacles presently due to its high cost. Therefore, in order to increase the rates of implantation, the improved assessment methodology was required. Accordingly, many different approaches have been suggested to reflect the embryo's function or physiological state. Several non-invasive methods assessing embryo viability and the potentiality of development have been established, such as proteomics (6), measurement of respiration rate (7), soluble human leukocyte antigen-G (8), pyruvate uptake (9) and glucose uptake (10). However, all of these methods have not been widely used in clinic because they were either expensive and required dedicated equipment, technical staff or do not provide results quickly enough to be used within the time frame of clinical IVF.

However, the Raman spectroscopy technique, used to observe vibration of bonds, rotational, and other lowfrequency modes in a system, has been commonly used in chemistry to provide a fingerprint by which molecules can be identified (11) and serves as an are of interest. Indeed, over the past few years, Raman spectroscopy has become a powerful diagnostic tool in the life sciences (12). Currently, in the field of assisted reproduction technology, there are few studies using the Raman technique to investigate the correlation between the metabonomics profile and the embryo quality as well as the developmental potential and the outcome of pregnancy. Seli et al reported that the non-invasive metabonomics profiling of embryo culture media using Raman and near infrared (NIR) spectroscopy were correlated with the pregnancy outcome in women undergoing IVF (13). They also carried out two studies demonstrating that metabonomics models developed using NIR or Raman may predict embryo viability $(14,15)$. The same group conducted an analogous 
Table I. Embryo assessment criteria.

\begin{tabular}{lccccccc}
\hline Classification & Grade & Cell no. & Fragmentation (\%) & Symmetry & Multi-nucleation & Vacuoles & Zona pellucida \\
\hline High quality & A & $4(\mathrm{~d} 2) \rightarrow 7-8(\mathrm{~d} 3)$ & $<10$ & Even & No & No & Normal \\
3-4 & B & $4(\mathrm{~d} 2) \rightarrow 7-8(\mathrm{~d} 3)$ & $11-25$ & Even & No & No & Normal \\
& & $4(\mathrm{~d} 2) \rightarrow \geq 9(\mathrm{~d} 3)$ & $11-25$ & Even & No & No & Normal \\
Poor quality & $\mathrm{C}$ & $2,4,6(\mathrm{~d} 2) \rightarrow>7(\mathrm{~d} 3)$ & $26-35$ & Uneven & No & Few & Abnormal \\
$4-5$ & & $6(\mathrm{~d} 2) \rightarrow>8(\mathrm{~d} 3)$ & $<35$ & Uneven & No & Few & Abnormal \\
& & 2 or $4(\mathrm{~d} 2) \rightarrow 6(\mathrm{~d} 3)$ & $<35$ & Uneven & No & Few & Abnormal \\
& & $3(\mathrm{~d} 2) \rightarrow>6(\mathrm{~d} 3)$ & $<35$ & Uneven & No & Few & Abnormal \\
\hline
\end{tabular}

prospective study assessing day 3 and 5 culture media which confirmed the strong association of the metabonomics profile and clinical outcome, high sensitivity as well as the specificity and accuracy of the profiling (15). Recently, these findings have been extended by Zhao et al who found that a combination of embryo morphology scores and Raman determined that sodium pyruvate and phenylalanine levels in culture medium were indicative of high reproductive potential (16). Although the abovementioned reports demonstrated that Raman spectroscopy was a potential technique for the assessment of the embryo quality, the results from the studies were not consistent for the diversity of the Raman machine, embryo culture media and the study protocol. The development and validation of the method of assessment for embryo quality with Raman spectroscopy in various reproductive centers is needed to prove the reliability of this assay method.

Thus, the current study aimed to assess the metabonomics profile of embryo culture media using Raman spectroscopy comparing with conventional morphologic methods. We also aimed to develop a rapid, non-invasive method for the assessment of the developmental potential of embryos.

\section{Materials and methods}

Patients and ethics approval. All the patients enrolled in our study were treated at the Department of Reproductive Medicine of Nantong Maternity and Child Health Hospital, in Nantong, China. Ethical approval for the protocol was received from the Medical Ethics Committee of Nantong Maternity and Child Health Hospital. Each couple whose embryos were enrolled into the study signed a written informed consent.

Patient treatment. Ten patients who underwent ovarian stimulation using either a long gonadotropin-releasing hormone (GnRH) agonist protocol or a flexible GnRH antagonist protocol were selected to our study. Oocyte retrieval was performed $36 \mathrm{~h}$ after the human chorionic gonadotropin injection by transvaginal ultrasound-guided aspiration. The oocyte cumulus complexes were isolated, the majority of the cumulus were stripped mechanically and the oocytes were placed into individual $25 \mu \mathrm{l}$ droplets of media (Quinn's advantage cleavage medium (SAGE In-Vitro Fertilization, Inc., Trumbull, CT, USA) supplemented with $10 \%$ serum substitute supplement (Irvine Scientific, Santa Ana, CA, USA). Fertilized oocytes were individually cultured for 3 days in $25 \mu 1$ pre-equilibrated drops of sequential culture media.
Embryo assessment. A standard embryo scoring system was used for the evaluation of the quality of embryo based on the rate of cleavage and morphology (17). The morphological criteria considered include: i) The number of pronuclei and polar bodies (zygotes); ii) cell number; iii) evenness of mitotic divisions; iv) presence of micronuclei; and v) amount of cellular fragmentation (cleavage embryos). The embryo grade A and B were defined as high-quality embryo and embryo grade $\mathrm{C}$ was considered as poor quality embryo (Table I). The same embryologist preformed all embryology and embryo scoring in this study to ensure the consistency.

Culture medium specimens. The embryo selection for implantation was based on embryo morphology at day 3 . Following embryo transfer, the spent culture media were collected individually and stored at $-80^{\circ} \mathrm{C}$. The equilibrated culture media without embryos was also collected and used for normalization. During each experimental step, laboratory personnel wore gloves and coat, and the physical isolation was guaranteed by working in clean air hoods.

Raman spectroscopy. The spent culture media were thawed at room temperature $\left(25^{\circ} \mathrm{C}\right)$ for $30 \mathrm{~min}$ before analysis. The mineral oil on the surface of the spent media was removed by capillary siphon until there was no visual stratification. After which the media were vortexed for $10 \mathrm{sec}$ and centrifuged for $10 \mathrm{~min}$ at $11,000 \mathrm{x} \mathrm{g}$. Next, $20 \mu \mathrm{l}$ of embryo culture medium, taken from the cryovials by pipette gun, were dropped into a round glass with a thin layer of gold film on the surface, and the glass was placed onto a slide. Ten points were selected and Raman spectroscopy was measured for each sample. Raman analysis was conducted using a Raman spectrometer (BWTEK; B\&W Tek, Inc. Newark, DE, USA). The spectra were recorded from 600 to $2,000 \mathrm{~cm}^{-1}$. The signal acquisition time was $60 \mathrm{sec}$. Raman spectroscopy was collected at $10-15$ points/sample. Laser Raman spectroscopy of the samples from 10 patients was conducted under the parameters above. The original spectra were preprocessed automatically by Origin 6.0 software.

\section{Results and Discussion}

Although numerous studies have verified the feasibility of metabonomics profiling of spent media to select the high quality embryo, the validation from other laboratories is needed for the consistency of results on the diversity of the 


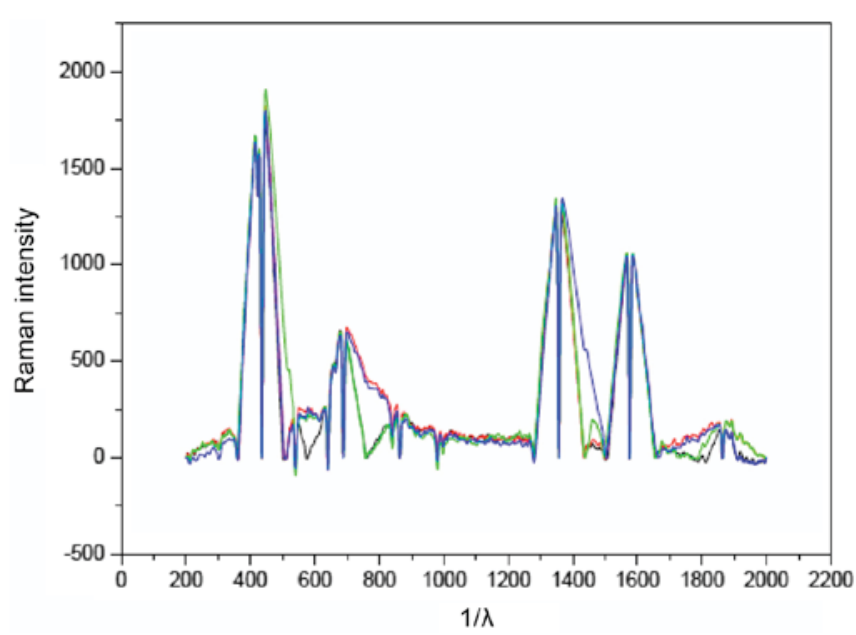

Figure 1. Typical Raman spectrum of spent embryo media. Green and black line: Raman spectrum of spent media of high quality embryo. Red and blue line: Raman spectrum of spent media of poor quality embryo. Statistical analyses were not applied due to the limited number of patients.

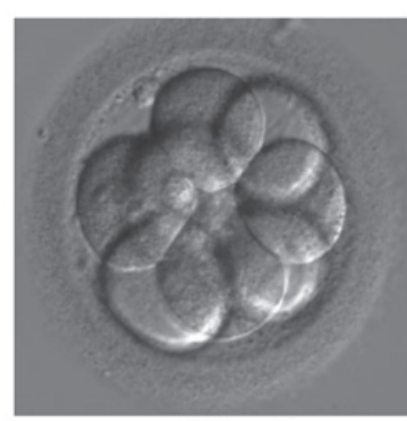

High quality

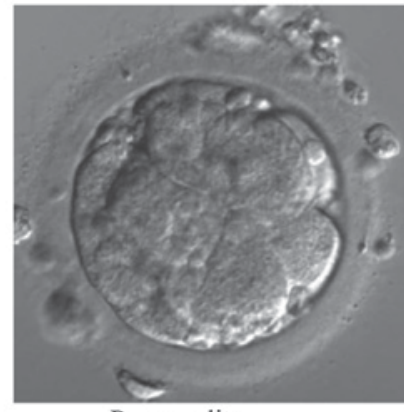

Poor quality
Figure 2. The image of embryos of day 3 in different grade by morphological scoring. The high quality with amplification is representative for the morphopathology in cells.

medium, Raman instrument and protocol of analysis. In the present study, we analyzed spent culture media of embryos using Raman spectroscopy, trying to evaluate the embryo, and compare with the conventional morphological methods. The IVF spent culture media were thawed at room temperature for $30 \mathrm{~min}$. The mineral oil layer on the surface, which act as the protecting shell to prevent moisture volatilizing were taken away with physical siphon until there was no visual stratification. Our preliminary results showed that there is no effect on spectral signals of embryo media for mineral oil. To verify the consistency of measurement, different grade embryos from the same couple were analyzed. Figs. 1 and 2 show the typical Raman spectrograms and microphotography of embryos of day 3 in different grade by morphological scoring, respectively. The peak area of at Raman shifting $755 \mathrm{~cm}^{-1}$ from the high quality embryo was obviously different to the poor one (Fig. 1). Raman shifting $750 \mathrm{~cm}^{-1}$ was the characteristic peak for tryptophan, an essential amino acid which cannot be synthesized by the organism and must be provided by the exterior nutrition (15). In the fresh media for embryo culture, tryptophan is $0.02 \mathrm{mM}$ in general. More tryptophan was consumed by the high quality embryo for their development comparing to the poor embryo. Thus, the metabolism of a healthy embryo may alter the surrounding environment differently from one that is less healthy and thus possessing less reproductive potential. It may produce a profile that may more clearly be associated with embryo viability.

Amino acids have numerous roles in early embryo development in addition to protein synthesis. Preimplantation embryos can consume and produce amino acids in a manner dependent on the stage of development that may be predictive of subsequent viability. Tryptophan has a variety of metabolic functions within the cell. It is incorporated into the polypeptide chains of enzymes and proteins, and it is a biosynthetic precursor of the cofactor NAD, the siderophore quinolobactin, and the neuron transmitters serotonin and melatonin (18).

Numerous studies have analyzed the remaining media to determine novel assessing and screening methods to select the greatest potential embryo $(16,19)$. Different from the former studies, the spent media from conventional IVF were analyzed with Raman spectroscopy in our research. Although to the best of our knowledge, there is no research or epidemiology study indicating that intracytoplasmic sperm injection could impair the embryo development and the pregnancy outcome to this day, the different protocol of fertilization may have led to the different metabolites. Thus, only the remaining conventional IVF media were collected and analyzed in this study.

There are several studies trying to understand the correlation between Raman spectrum of spent media and the outcome of pregnancy of a woman implanted with the embryo (20). It is clear that the quality of the embryo is not an independent factor contributing to the pregnancy outcome. There are other reasons which may impair the outcome of the embryo transfer, such as the endometrium situation, psychological state, or hormone level. Therefore, we only focused on the correlation between the Raman spectrograms and morphological grading. In the present study, we compared the metabolic profile with the morphological classification. However, we did not calculate the correlation index, because of the tiny number of samples in the study. In subsequent investigations, we intend to include a larger number of samples.

In conclusion, the profile of Raman spectrum of the spent embryo culture media was presented comparing the morphological assessment in our study. Without any doubt, an appropriate evaluation method can improve the rates of embryo implantation and pregnancy. To substantiate the results in our study, additional investigations of the correlation between the Raman spectrum and rate of pregnancy should be conducted in the near future.

\section{Acknowledgements}

The study was supported by the Jiangsu Maternity and Child Health Research Project (F201347) and the Nantong Science and Technology Project (HS2013012).

\section{References}

1. Ombelet W, Cooke I, Dyer S, Serour G and Devroey P: Infertility and the provision of infertility medical services in developing countries. Hum Reprod Update 14: 605-621, 2008.

2. Ying LY, Wu LH and Loke AY: The experience of Chinese couples dndergoing in vitro fertilization treatment: perception of the treatment process and partner support. PLoS One 10: e0139691, 2015. 
3. Nasiri $\mathrm{N}$ and Eftekhari-Yazdi P: An overview of the available methods for morphological scoring of pre-implantation embryos in in vitro fertilization. Cell J 16: 392-405, 2015.

4. Santos Filho E, Noble JA, Poli M, Griffiths T, Emerson G and Wells D: A method for semi-automatic grading of human blastocyst microscope images. Hum Reprod 27: 2641-2648, 2012.

5. Kovacs P: Embryo selection: the role of time-lapse monitoring. Reprod Biol Endocrinol 12: 124, 2014.

6. Nyalwidhe J, Burch T, Bocca S, Cazares L, Green-Mitchell S, Cooke M, Birdsall P, Basu G, Semmes OJ and Oehninger S: The search for biomarkers of human embryo developmental potential in IVF: a comprehensive proteomic approach. Mol Hum Reprod 19: 250-263, 2013.

7. Wilding M, Dale B, Marino M, di Matteo L, Alviggi C, Pisaturo ML, Lombardi L and De Placido G: Mitochondrial aggregation patterns and activity in human oocytes and preimplantation embryos. Hum Reprod 16: 909-917, 2001.

8. Vercammen MJ, Verloes A, Van de Velde H and Haentjens P: Accuracy of soluble human leukocyte antigen-G for predicting pregnancy among women undergoing infertility treatment: meta-analysis. Hum Reprod Update 14: 209-218, 2008.

9. Ray PF, Conaghan J, Winston RM and Handyside AH: Increased number of cells and metabolic activity in male human preimplantation embryos following in vitro fertilization. J Reprod Fertil 104: 165-171, 1995.

10. Gardner DK, Wale PL, Collins R and Lane M: Glucose consumption of single post-compaction human embryos is predictive of embryo sex and live birth outcome. Hum Reprod 26: 1981-1986, 2011.

11. Votteler M, Carvajal Berrio DA, Pudlas M, Walles H and Schenke-Layland K: Non-contact, label-free monitoring of cells and extracellular matrix using Raman spectroscopy. J Vis Exp 63: 3977, 2012.

12. Brauchle E and Schenke-Layland K: Raman spectroscopy in biomedicine - non-invasive in vitro analysis of cells and extracellular matrix components in tissues. Biotechnol J 8: 288-297, 2013
13. Seli E, Vergouw CG, Morita H, Botros L, Roos P, Lambalk CB, Yamashita N, Kato O and Sakkas D: Noninvasive metabolomic profiling as an adjunct to morphology for noninvasive embryo assessment in women undergoing single embryo transfer. Fertil Steril 94: 535-542, 2010.

14. Seli E, Sakkas D, Scott R, Kwok SC, Rosendahl SM and Burns DH: Noninvasive metabolomic profiling of embryo culture media using Raman and near-infrared spectroscopy correlates with reproductive potential of embryos in women undergoing in vitro fertilization. Fertil Steril 88: 1350-1357, 2007.

15. Scott R, Seli E, Miller K, Sakkas D, Scott K and Burns DH: Noninvasive metabolomic profiling of human embryo culture media using Raman spectroscopy predicts embryonic reproductive potential: a prospective blinded pilot study. Fertil Steril 90: 77-83, 2008.

16. Zhao Q, Yin T, Peng J, Zou Y, Yang J, Shen A and Hu J: Noninvasive metabolomic profling of human embryo culture media using a simple spectroscopy adjunct to morphology for embryo assessment in in vitro fertilization (IVF). Int J Mol Sci 14: 6556-6570, 2013.

17. Alpha Scientists in Reproductive Medicine and ESHRE Special Interest Group of Embryology: The Istanbul consensus workshop on embryo assessment: proceedings of an expert meeting. Hum Reprod 26: 1270-1283, 2011.

18. Radwanski ER and Last RL: Tryptophan biosynthesis and metabolism: biochemical and molecular genetics. Plant Cell 7: 921-934, 1995.

19. Telford NA, Watson AJ and Schultz GA: Transition from maternal to embryonic control in early mammalian development: a comparison of several species. Mol Reprod Dev 26: 90-100, 1990.

20. Chen JY, Schopf JW, Bottjer DJ, Zhang CY, Kudryavtsev AB, Tripathi AB, Wang XQ, Yang YH, Gao X and Yang Y: Raman spectra of a Lower Cambrian ctenophore embryo from southwestern Shaanxi, China. Proc Natl Acad Sci U S A 104: 6289-6292, 2007. 